\title{
Terahertz wave propagation characteristics of multi- modes on complexity microstrip-slot coupled lines
}

\author{
Jing Zhu and Dan Zhang ${ }^{\text {a) }}$ \\ College of Information Science and Technology, Nanjing Forestry University, \\ Nanjing 210037, China \\ a)zhangdan@njfu.edu.cn
}

\begin{abstract}
The Terahertz $(\mathrm{THz})$ technology field is currently a popular scientific topic. In this paper, the transmission characteristics of two coupled planar transmission lines (microstrip-double slot and double microstrip-slot coupled lines) are investigated via odd-mode excitation and even-mode excitation in the THz band. Furthermore, we study the attenuation constant and the effective refractive index of the two transmission lines under investigation. We have compared the propagation characteristics of $\mathrm{THz}$ pulses on microstrip-lines and coplanar strip-lines; we find very good agreement between the observed results.
\end{abstract}

Keywords: terahertz, microstrip, slotline, even mode, odd mode

Classification: Microwave and millimeter-wave devices, circuits, and modules

\section{References}

[1] I. Hosako, et al.: "At the dawn of a new era in terahertz technology," Proc. IEEE 95 (2007) 1611 (DOI: 10.1109/JPROC.2007.898844).

[2] P. H. Siegel: "Terahertz technology," IEEE Trans. Microw. Theory Techn. 50 (2002) 910 (DOI: 10.1109/22.989974).

[3] M. B. Byrne, et al.: "Terahertz vibrational absorption spectroscopy using microstrip line waveguides," Appl. Phys. Lett. 93 (2008) 182904 (DOI: 10. 1063/1.3013349).

[4] Y. Kadoya, et al.: "THz wave propagation on strip lines: Devices, properties, and applications," Radio Eng. 17 (2008) 48.

[5] M. Y. Frankel, et al.: "Terahertz attenuation and dispersion characteristics of coplanar transmission lines," IEEE Trans. Microw. Theory Techn. 39 (1991) 910 (DOI: 10.1109/22.81658).

[6] A. Tsuchiya and H. Onodera: "Impact of radiation loss in on-chip transmissionline for terahertz applications," 16th IEEE Workshop Signal and Power Integrity (SPI) (2012) 125 (DOI: 10.1109/SaPIW.2012.6222926).

[7] Z. Chen: Fundamentals and Applications of Microwave Technology (Beijing University of Posts and Telecommunications Press, Beijing, 2002) 95.

[8] C.-P. Chen, et al:: "Design of pseudo-elliptical wideband bandpass filter using stub loaded short-circuited parallel-coupled three-line units," IEICE Trans. Electron. E93-C (2010) 1022 (DOI: 10.1587/transele.E93.C.1022).

[9] C.-P. Chen, et al.: "Design of a wideband filter with attenuation poles using a 
novel parallel-coupled three-line unit based on cross-coupling," IEICE Trans. Electron. E97-C (2014) 689 (DOI: 10.1587/transele.E97.C.689).

[10] C. Zhao and C. Zhang: Microwave Technology (Higher Education Press, Beijing, 2007) 87.

\section{Introduction}

Currently, terahertz (THz) technology is one of the more popular scientific research fields in many countries around the world [1]. Due to the special nature of the $\mathrm{THz}$ frequency band, $\mathrm{THz}$ waves show a series of unique special properties [2]. To meet the plane integration requirements of $\mathrm{THz}$ systems, several planar transmission lines have also been proposed for the transmission of THz waves. Planar structures like microstrip lines [3], strip lines [4], slot lines, and coplanar waveguides [5, 6] are often used in low-cost designs.

To reduce size characteristics, more and more designers use double-layer structures in their designs because both sides of the dielectric substrate are used. Microstrip and slot lines are the most common planar transmission lines [7]. When microstrip line is combined with slot lines, they form microstrip-slot line conversion structures. Microwave-based integrated designs are often freely-used in these structures. The coupling between them can be used to form filters [8, 9], directional couplers and other elements [10]. In most cases, a microstrip-slot line coupling structure is used to transmit signals between the two faces of a dielectric substrate.

In this paper, we analyze the transmission characteristics of the coupling transmission lines using HFSS soft that is based on finite element method (FEM). Cross-sectional views of the two coupling lines are shown in Fig. 1. The transmission lines on the upper and bottom surface of the dielectric substrate, whose thickness $h$ is $20-\mu \mathrm{m}$, uses gold with a thickness $t=0.2 \mu \mathrm{m}$. The structure of the microstrip-double slot coupled line is to fabricate a microstrip line on one side of the polyimide substrate and a coplanar waveguide on the other side (Fig. 1(a)). The structure of the double microstrip-slot coupled line is fabricated using two microstrip lines on one side of the quartz-glass substrate and a slot line on the other side (Fig. 1(b)).

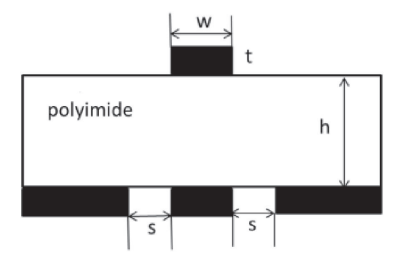

(a)

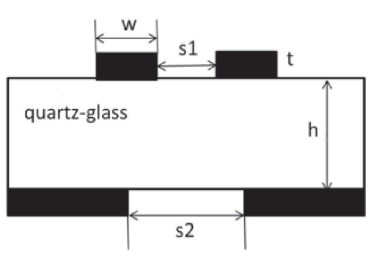

(b)

Fig. 1. The cross-sectional view of the transmission lines of a (a) microstrip-double-slot coupled line and a (b) double microstripslot coupled line are shown.

\section{Microstrip line (MSL) and coplanar stripline (CPS)} strip-lines and coplanar strip-lines are studied and compared using [4] as the 
baseline. The attenuation constant and the effective index-of-refraction of MSL and CPS are plotted as functions of frequency in Figs. 2-3. In Fig. 3, the squares, diamonds, and circles correspond to the sapphire, quartz, and polymer substrates, respectively. The dotted-dash, dash, and solid lines correspond to the sapphire, quartz, and polymer substrates, respectively. Our results agree with the predictions based on the analytical formula used in microwave regime [4].

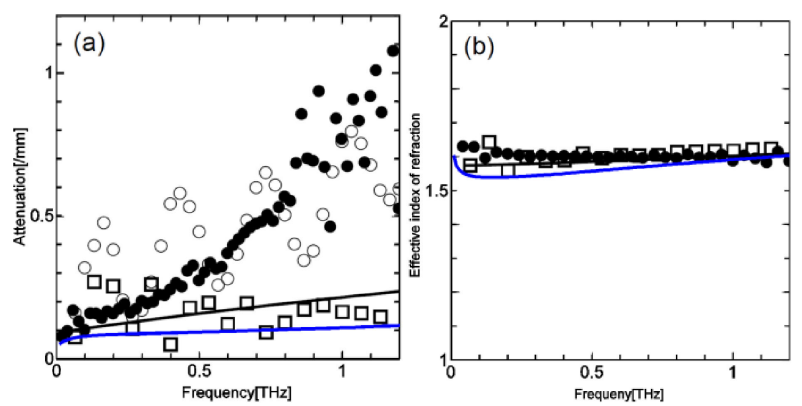

Fig. 2. The (a) attenuation constant and (b) effective index-ofrefraction associated with the propagation of the $\mathrm{THz}$ pulses along the MSL are shown. The solid circles and open squares represent the experimental and FDTD results, respectively. The black solid line and the open circles in (a) are the values estimated using the analytical expressions used in the microwave regime with the measured conductance of the metal line and the tangent of the polyimide film $(\tan \delta)$. The black solid line in (b) is a prediction based on a model in microwave regime. The blue lines are our results.
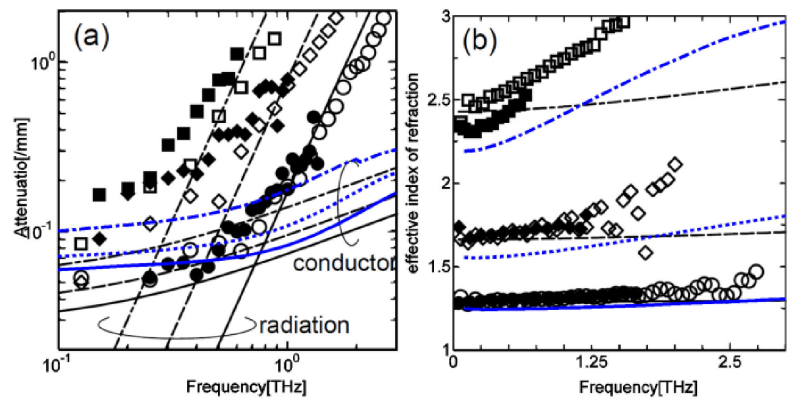

Fig. 3. The (a) attenuation constant and (b) effective index-of-refraction associated with the propagation of THz pulses along the CPS are shown. The solid and open symbols represent the experimental and the FDTD results, respectively. The black lines are the predictions, which are based on an analytical formula used in the microwave regime. The blue lines are our results.

\section{Microstrip-double slot coupled line}

In this section, we analyze the propagation characteristics of $\mathrm{THz}$ pulses on microstrip-double slot coupled lines. The attenuation and the effective index-ofrefraction are plotted as functions of frequency in Fig. 4. We find that, for the odd-mode excitation case, the attenuation and effective index are lower than the attenuation and effective index for the even-mode excitation case. Figs. 5-8 show the transmission characteristics using a frequency of 3-THz. In the even-mode excitation case, the effective refractive index increases and the attenuation constant decreases with $w$, but $s$ has no obvious influence on the effective refractive index or the attenuation constant, which resembles the transmission characteristics in a 
microstrip line (Figs. 5 and 6). As shown in Figs. 7 and 8, the effective refractive index and attenuation constant decrease with increasing $s$ in the odd-mode excitation case, but odd-mode excitations are less sensitive to the presence of the strip, which resembles the transmission characteristics in a slot line. This may be because most of the energy in this mode is confined to an area near a slot.
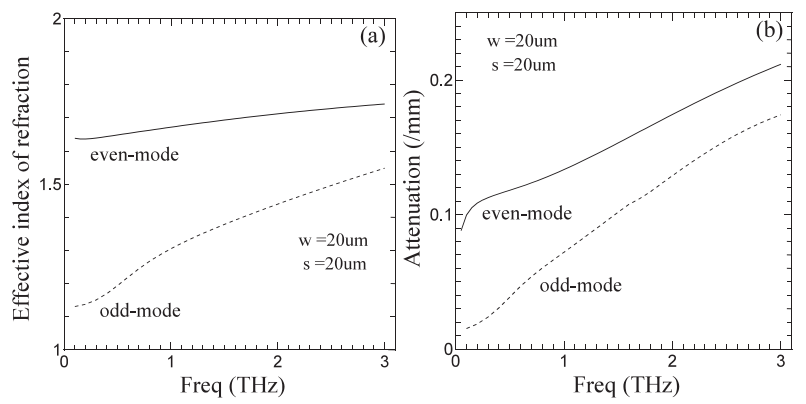

Fig. 4. The (a) effective refractive index and (b) attenuation constant associated with the propagation along the microstrip-double slot coupled line are shown.
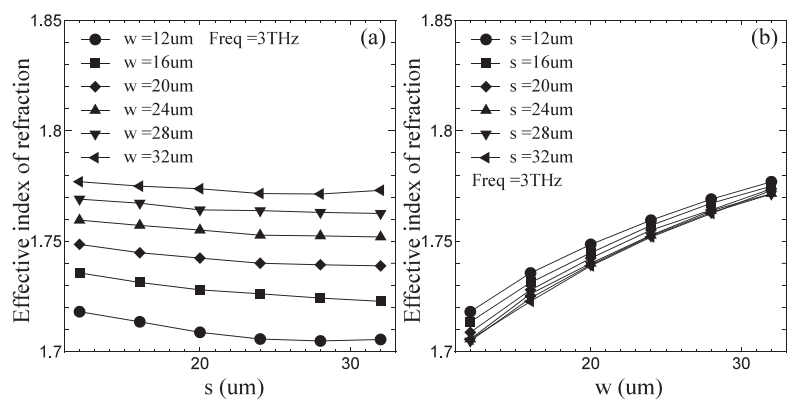

Fig. 5. The variation of the effective refractive index with $s$ and $w$ in the even-mode excitation case.
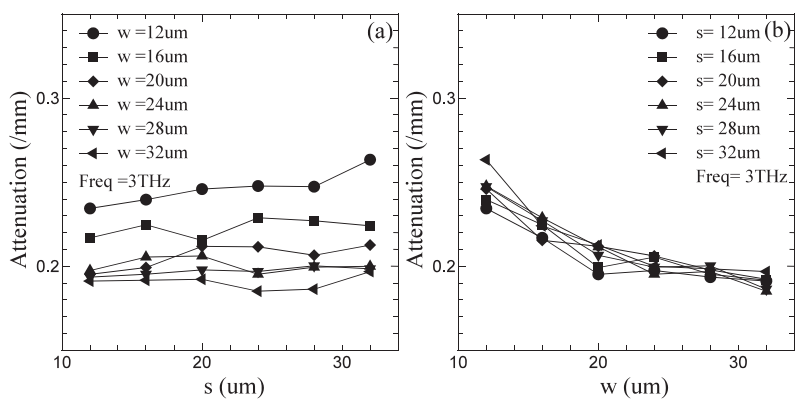

Fig. 6. The variation of the attenuation constant with $s$ and $w$ in the even-mode excitation case.

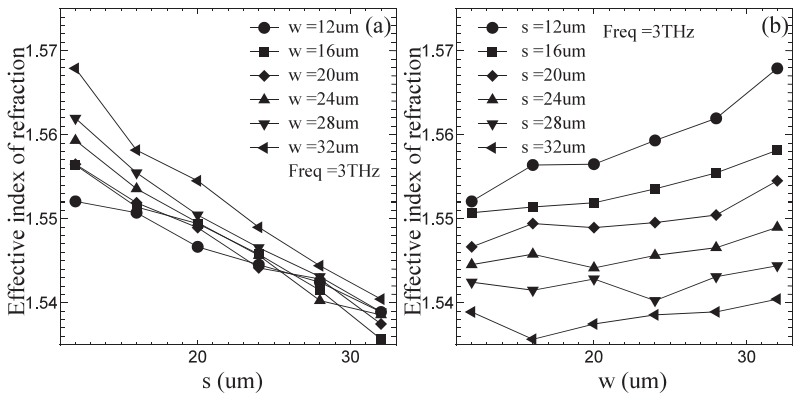



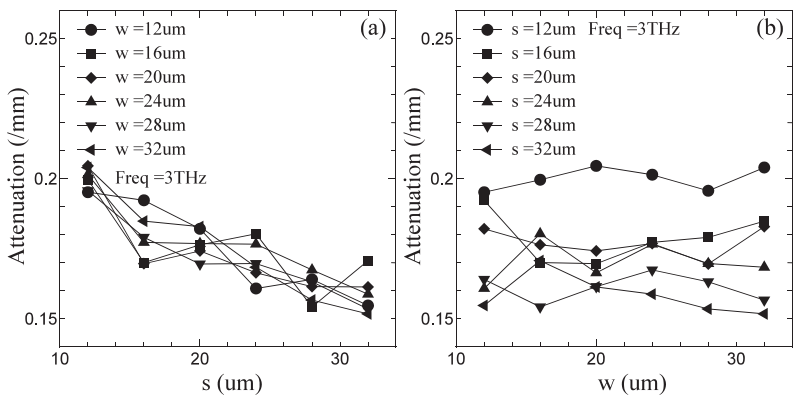

Fig. 8. The variation of attenuation constant with $s$ and $w$ in the oddmode excitation case.

\section{Double microstrip-slot coupled line}

In Fig. 9, we show plots of the attenuation constant and the effective refractive index as a function of frequency. The effective index-of-refraction, with odd-mode excitation, is lower than effective index-of-refraction with even-mode excitation; this is likely because the attenuation constant with odd-mode excitation is higher than the attenuation with even-mode excitation.
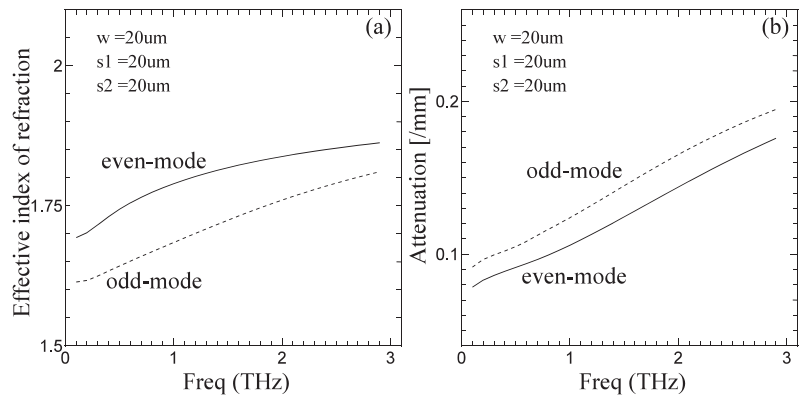

Fig. 9. The (a) effective index-of-refraction and the (b) attenuation constant associated with the propagation along the double microstrip-slot coupled line is shown.
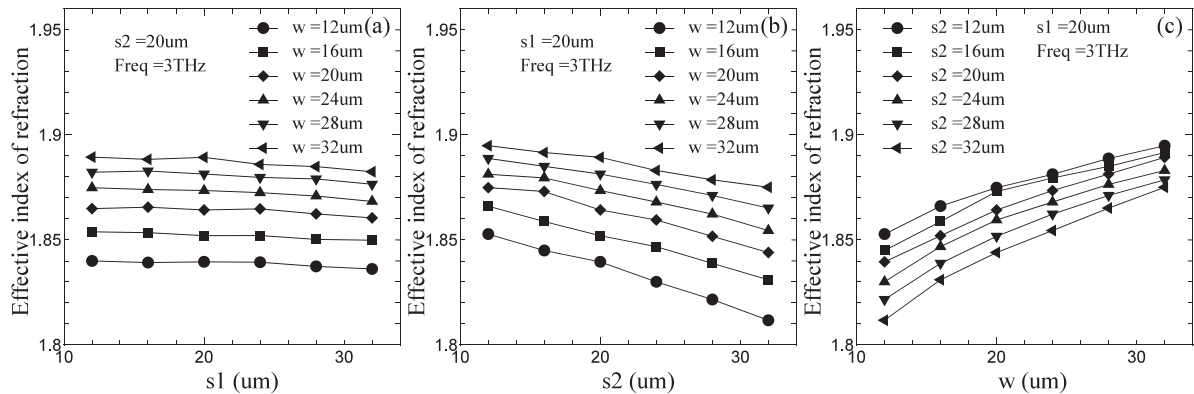

Fig. 10. The variation of effective refractive index with (a) $s_{1}$, (b) $s_{2}$ and (c) $w$ in the even-mode excitation case is shown.
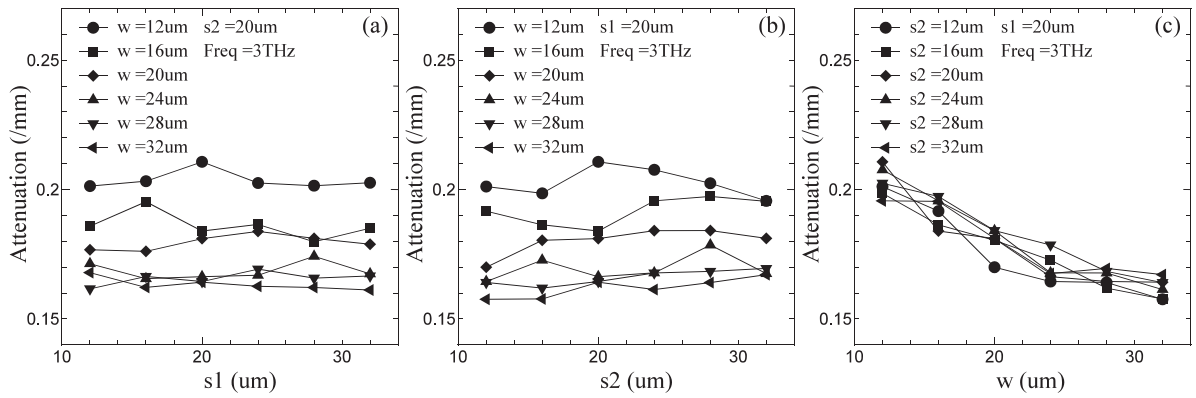

Fig. 11. The variation of the attenuation constant with (a) $s_{1}$, (b) $s_{2}$ and (c) $w$ in the even-mode excitation case. 
The transmission characteristics in the $3-\mathrm{THz}$ frequency band are shown in Figs. 10-13. As shown in Figs. 10 and 11, $s_{1}$ has no influence on the effective refractive index and attenuation constant, in the even-mode excitation case; this means that the coupling between the two strips has little effect on the transmission characteristics. The effective refractive index increases with $w$ but decreases with $s_{2}$, and attenuation constant decreases with w. In Figs. 12 and 13, in the odd-mode excitation case, when $s_{1}$ is bigger than $20 \mu \mathrm{m}$, the effective refractive index starts increasing with $s_{1}$; this means that the coupling between the two strips start exhibiting effects when $s_{1}$ is greater than $20 \mu \mathrm{m}$. The effective refractive index increases with $w$, while the attenuation constant decreases with $w$. In contrast, transmission characteristics aren't affected by $s_{2}$.
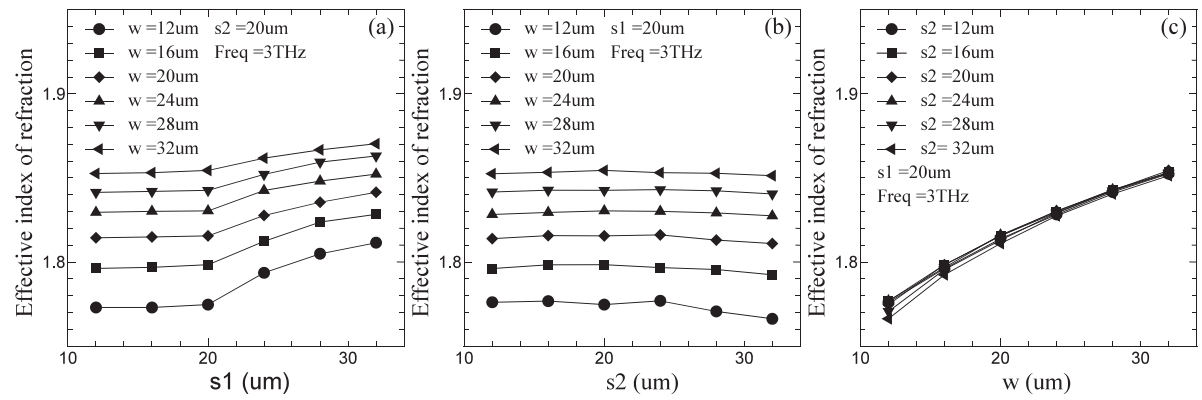

Fig. 12. The variation of the effective refractive index with (a) $s_{1}$, (b) $s_{2}$ and (c) $w$ in the odd-mode excitation case.
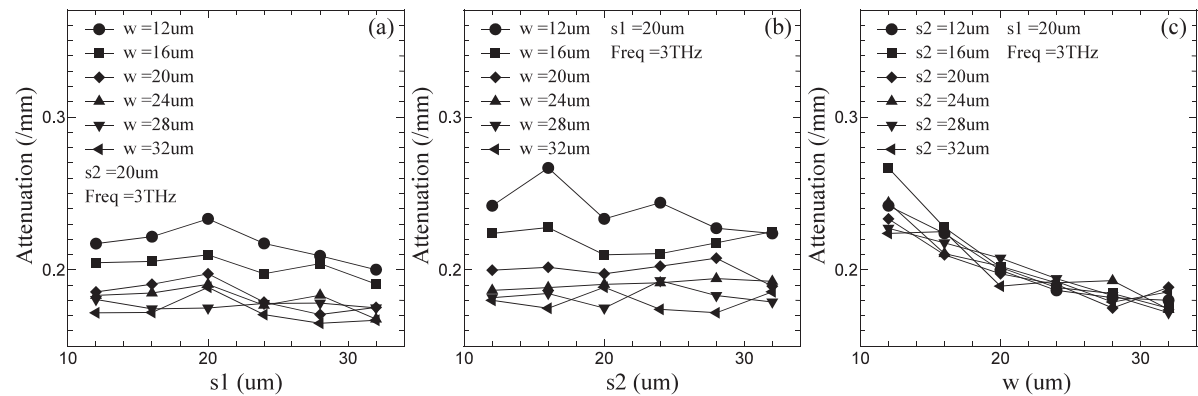

Fig. 13. The variation of the attenuation constant with (a) $s_{1}$, (b) $s_{2}$ and (c) $w$ in the even-mode excitation case.

\section{Conclusion}

In this paper, we investigate the propagation of $\mathrm{THz}$ pulses on a microstrip-double slot coupled line and double microstrip-slot coupled line for even- and odd-mode excitations. The measurements we collected agree with the results in the reference [4], which confirms that our method is correct. For microstrip-double slot coupled lines, the attenuation constant with odd-mode excitation are lower than it with even-mode excitation. The $w$ has an obvious influence on transmission characteristics in the even-mode excitation case, while the $s$ has an obvious influence on the transmission characteristics in the odd-mode excitation case for the $3 \mathrm{THz}$ band. For double microstrip-slot coupled lines, the attenuation constant in the even-mode excitation is lower than the attenuation constant in the odd-mode excitation case. The $w$ has an obvious influence on the transmission characteristics in both the evenmode and odd-mode excitations in the $3 \mathrm{THz}$ band. 